\title{
Physicians and AIDS care: does knowledge influence their attitude and comfort in rendering care?
}

\author{
Adetoyeje $\mathrm{Y}^{1}$. Oyeyemi, Bashir O. Oyeyemi², Ibrahim S. Bello², \\ College of Health Related Professions, State University of New York at Brooklyn, 450 Clarkson Avenue, Brooklyn, \\ New York, USA. 2. Department of Pediatrics, Federal Medical Center, Katsina, Nigeria. 3. Department of General \\ Medical Practice, Obafemi Awolowo University Teaching Hospital Complex, Ile-Ife, Nigeria.
}

Address all correspondents to Dr. A.Y. Oyeyemi, Dr. Susan Smith McKinney Nursing \& Rehabilitation Center, 594 Albany Avenue, Brooklyn, NY 11203;Telephone (718) 270 7216; e-mail: oyeyemia@nychhc.org

\section{SUMMARY}

\begin{abstract}
Adequate knowledge, positive attitude, and feeling of comfort are important factors in providing compassionate care to patients. The purpose of this study was to assess physicians' knowledge, attitude and global comfort in caring for patients with AIDS (PWA), to determine the sociodemographic variables that could influence physicians' attitude and global comfort, and to identify any relationship between their knowledge, attitude and comfort. Consultants and residents $(\mathrm{N}=211)$ in two Nigerian teaching hospitals were surveyed using a two-part questionnaire. Part I elicited sociodemographic and previous AIDS encounter information, and Part II assessed knowledge, attitude and global comfort with AIDS patients care. Nigerian physicians showed satisfactory knowledge, but they harbored negative attitude and low level of comfort in caring for PWA. Previous AIDS care experience, age and being a consultant or a senior resident influenced attitude, while male gender and knowing someone with AIDS influenced global comfort. Knowledge is weakly but positively associated with attitude, while attitude is modestly associated with comfort. The study reinforced the need for an ongoing education focused on experiential learning, and professional socialization in order to influence physicians' attitude and enhance their feeling of comfort when caring for PWA.
\end{abstract}

[Afr J Health Sci. 2007;14:37-43]

\section{Introduction}

Adequate knowledge, positive attitude, and not feeling uncomfortable in administering a treatment are important factors in providing compassionate care to patients. Poor attitude could debar the necessary application of logic and scientific method in the practice of medicine, and could also result in fragmented care, apathy and discomfort during care, with potential impact on outcome optimization. Unlike factual knowledge acquired through didactic, attitudes are biases and stereotypes and could only be mediated through the socialization process during training [1]. It is plausible that physicians' feeling of comfort treating patients with AIDS (PWA) could not only be influenced by their knowledge of the condition, but also by their attitude shaped by the prevalent societal stigma, fear, and assumed multisystem risks attributed to AIDS.

Many previous studies have investigated physicians' knowledge, beliefs, fears, and attitudes to
AIDS [2-9], and their willingness or degree of comfort in providing care to PWA[4-6] In the extant literature, physicians' factual knowledge on AIDS have been described as good or high [5-8], while other studies reported lack of knowledge [9], inadequate knowledge [10], and knowledge gap among physicians [3-4]. Physicians were fearful of AIDS [10], harbored negative attitude [2], and have judgmental evaluations against PWA [10].

Compared to a decade ago, much insight has been gained into the pathology of the human immunodeficiency virus and public awareness has grown worldwide, following consistent campaigns against AIDS epidemics. The varied level of knowledge reported in the above studies could therefore be attributed to the different measuring tools and varying foci of the studies, conducted at different time period in AIDS awareness. 


\section{Purpose of the study}

The primary purpose of this study was (1) to assess Nigerian physicians' knowledge, attitude and global comfort with AIDS care, (2) to identify the sociodemographic variables that may influence physicians' attitude and global comfort with AIDS care, and (3) to identify any relationship between knowledge, attitude and comfort in rendering care to PWA.

\section{Materials and Methods \\ Sample}

Two hundred and eleven physicians (168 males and 43 females) practicing in two teaching hospitals, located in urban areas in two contiguous states of Kwara and Osun in Nigeria, participated in the study. In order of professional ranking, subjects were consultants, residents (senior registrars, registrars, senior house officers) and house officers at Obafemi Awolowo University Teaching Hospital (OTH) and University of Ilorin Teaching Hospital (UTH).

\section{Instruments}

A two-parts 95-item questionnaire was designed to answer questions posed in the study. Part 1 of the questionnaire solicited sociodemographic information such as age, gender, marital status, rank, specialty and religion. Respondents were asked about their previous experience with AIDS education and patient care. They were asked whether they know any family member or any person with AIDS, ever been asked, ever cared or ever refused care to PWA, and whether they are willing to provide care to these patients. Possible responses were "Yes" and "No". Furthermore, they were asked about any previous AIDS instruction, the mode, effectiveness, duration, and how satisfied they were with the instructions received.

Part II of the questionnaire comprised three subscales that evaluated respondents' knowledge, attitudes and global comfort on AIDS. Subscale 1 consisted of 34 questions on pathophysiology, universal precaution, prevention and treatment of AIDS, and were framed to elicit "Yes", "No", or "Not sure" responses. Only the correct answers were counted and 'Not Sure' responses were not given any credit. The minimum and maximum scores on the subscale were 0 and 34 respectively, with a high score indicating good factual knowledge on AIDS. Subscale 2 consisted of 35 questions designed to assess respondents' attitudes. Responses were rated on a five-point Likert scale ranging from Strongly Agree (1) to Strongly Disagree (5). The minimum and maximum scores were 35 and 175 , respectively. Questions on attitude subscale were worded negatively. Therefore, the higher the subjects' total score, the more positive their attitude towards PWA.

Subscale 3 consisted of 10 -item questions on common medical examination and procedures and on exercise and physiotherapy referral. Participants were asked as to their level of comfort or discomfort in performing procedures such as rectal examination, vaginal examination, surgery, setting arterial line, lumbar puncture, venepuncture and mouth-to- mouth resuscitation on PWA. They were also asked whether they would not wear gloves to casually handle someone with HIV/AIDS, and who has no open wound, whether they would recommend regular exercise, and whether they would refer AIDS patients for physiotherapy. Respondents were asked to rate their level of comfort on a five-point Likert scale from "Very Uncomfortable" (1) to "Very Comfortable" (5)

Subscales 1 and 2 were originally developed by Held [11], and adapted by Balogun et al. [12]. Held [11] reported reliability coefficients of 0.93 and 0.82 for the knowledge and attitude subscales respectively (subscales I and 2), tested in one-week interval. In a separate study, Balogun et al. [12] reported correlation coefficient of 0.68 and 0.83 respectively indicating moderate reproducibility with a test retest interval of five weeks. Five randomly selected physicians across five specialty areas in one of the teaching hospitals agreed on the face validity of subscale 3 for assessing global comfort in caring for HIV infected patients.

\section{Procedure}

Using a departmental rank ordered list, every second physician was sampled in September 2003, by placing questionnaires in their mail pigeonholes located in the departmental offices. A total of 224 and 244 consultants, residents and house officers were on record at the UTH and OTH respectively. Questionnaires were also distributed during departmental rounds to reach residents and house officers with no mail pigeonholes. Subjects were asked to complete only one questionnaire. The physicians were informed in a cover letter accompanying the questionnaires that completion of the questionnaires implied consent to participate, and anonymity was assured. No name or any identification was required on the questionnaires. The ethics committees in both institutions approved this study.

In September 2003, one hundred and twenty-six questionnaires were distributed in UTH and 133 in OTH. Within six weeks of distribution, 98 and 113 of these questionnaires were returned completed from UTH and OTH respectively, giving a combined response rate of $81 \%$. The minimum female sample 
size that could ensure no more than plus or minus 0.10 sampling error at a confidence level of $95 \%$, for the physician population comprising $11.3 \%$ females was 38 [13]. A female sample size of 43 was therefore representative of the population. For each completed questionnaire, we determined the number of correct responses on the knowledge subscale. Similarly, we summed the ratings separately for subscales 2 and 3.

\section{Statistical Analysis}

Data analysis was accomplished using Analyse-It Statistical Software (Analyze-It Ltd, UK). Descriptive statistics was computed for all items. Analysis of variance was used to determine the influence of demographic variables (i.e. gender, rank, specialty and religion) on knowledge, attitude and level of comfort among the subjects, while group differences were explored with student t-test and Mann-Whitney U-test, using an alpha level of 0.05 .

\section{Results}

The mean age of the physicians was $32(\mathrm{SD}=6.0)$. One hundred and sixty-eight $(79.6 \%)$ of the subjects were males and $43(20.4 \%)$ were females. One hundred and twenty $(57.4 \%)$ of the subjects were married, and eighty-nine (42.6\%) were single. Subjects were Muslims (70.2\%), Christians (27.0\%) and few $(2.8 \%)$ were not affiliated to Islam or Christianity. Some selected demographic characteristics of the physicians are presented in Table 1. Participants were from across specialties including pediatrics, general medicine, obstetrics and gynecology, orthopedics, general surgery, psychiatry, ear nose and throat, family practice and anaesthesiology.

Table 1: Demographic characteristics of the physicians $(\mathrm{N}=211)$

\begin{tabular}{llcc}
\hline Variable & & $\mathrm{n}$ & $\%$ \\
\hline \multirow{2}{*}{ Children } & & & \\
& Yes & 85 & 40.3 \\
& No & 126 & 59.7 \\
& & \\
Rank & & 18 & 8.5 \\
& Consultant & 27 & 12.8 \\
& Senior registrar & 55 & 26.1 \\
& Registrar & 18 & 8.5 \\
Senior House Officer & 93 & 44.1 \\
House Officer & & \\
& Orthopedics & 15 & 7.1 \\
& General Surgery & 28 & 13.3 \\
& Obstetrics/Gynecology & 37 & 17.5 \\
& General Medicine & 34 & 16.1 \\
& Pediatrics & 46 & 21.8 \\
& Others & 51 & 24.2
\end{tabular}

Table 2 shows participants' responses to questions on prior encounter with AIDS. Overwhelming majority of the respondents had previously been asked to care for PWA, have never refused to care for AIDS patients, and were willing to care for PWA. Majority $(62.6 \%)$ of the respondents knew a family member or another person with AIDS. Overwhelming majority $(90.5 \%)$ had received instructions on AIDS, and were satisfied or somewhat satisfied with the instructions received $(83.4 \%)$. The most effective mode of instructions reported by the respondents was audiovisual (34.6\%), followed by lecture (32.3\%). Clinical observation, group discussion and selected reading were reported as most effective by $17.3 \%$, $13.5 \%$, and $2.3 \%$ of the subjects respectively. As a group, the mean knowledge scores was 27.0 (SD 
2.7), reflecting overall moderate level of knowledge on AIDS among physicians. Subjects' score ranged from 19-32, out of a total possible score of 34. The mean attitudes score was $110.6(\mathrm{SD}=22.2)$ out of a possible score of 175 . This score showed that the cohort of Nigerian physicians harbored negative attitude about AIDS. The mean global comfort score was $31.5(\mathrm{SD}=7.5)$ out of a total possible score of 50 , which showed that these physicians were neither comfortable nor uncomfortable caring for PWA.

Table 2: Physicians' perception, experience and previous instructions about providing care to patients with AIDS

\begin{tabular}{|c|c|c|c|c|c|c|}
\hline \multirow[t]{2}{*}{ Independent Variable } & \multicolumn{2}{|l|}{ Yes } & \multicolumn{2}{|c|}{ No } & \multicolumn{2}{|c|}{ Undecided } \\
\hline & $\mathrm{n}$ & $\%$ & $\mathrm{n}$ & $\%$ & $\mathrm{n}$ & $\%$ \\
\hline $\begin{array}{l}\text { Ever been asked to provide } \\
\text { services for PWA }\end{array}$ & 191 & 90.5 & 20 & 9.5 & & \\
\hline $\begin{array}{l}\text { Ever refused to provide } \\
\text { services for PWA }\end{array}$ & 201 & 95.3 & 10 & 4.7 & & \\
\hline Ever cared for PWA & 198 & 93.8 & 13 & 6.2 & & \\
\hline $\begin{array}{l}\text { Know of family member or } \\
\text { PWA }\end{array}$ & 132 & 62.6 & 79 & 37.4 & & \\
\hline Willing to care for PWA & 173 & 82.0 & 4 & 1.9 & 34 & 16.1 \\
\hline $\begin{array}{l}\text { Ever received instructions on } \\
\text { AIDS }\end{array}$ & 191 & 90.5 & 20 & 9.5 & & \\
\hline $\begin{array}{l}\text { Satisfied with instructions on } \\
\text { AIDS }\end{array}$ & $176^{\wedge}$ & 83.4 & 35 & 16.6 & & \\
\hline
\end{tabular}

PWA denotes patients or persons (living) with AIDS.

${ }^{\wedge} \mathrm{N}$ value is a total of respondents who were either satisfied or somewhat satisfied.

Data was analyzed with one way-ANOVA to ascertain differences by demographic characteristics, prior encounter with AIDS and by scores on knowledge, attitude and global comfort. No difference was observed by ranks. However, when respondents were dichotomized, significant difference $(\mathrm{P}<0.05)$ in mean age was found between the consultants and registrars (Mean=34.5 SD 6.5) on the one hand, and the house officers (Mean $=29.2 \mathrm{SD}$ 3.8 ) on the other hand. Table 3 showed physicians' knowledge, attitude and global comfort. Significant difference $(\mathrm{P}<0.05)$ in knowledge score was found between physicians who indicated their willingness to care for PWA, and those who did not or were undecided.

Those who answered "Yes" to the question "have you ever been asked to care for PWA" scored significantly higher $(\mathrm{P}<0.01)$ on attitude than those who answered "No" to the question. Significantly higher $(\mathrm{P}<0.01)$ attitude score was also found among those who answered "Yes" to the question "Are you willing to care for PWA", compared to those who answered "No" or were undecided. Significant difference $(\mathrm{P}<.05)$ in attitude was observed between consultants, senior registrars and registrars on the one hand, and senior house officers and house officers on the other hand (115.6 $\mathrm{SD}=21.3$ vs $106.3 \mathrm{SD}=22.4)$.

Male physicians scored significantly higher $(\mathrm{P}<.05)$ on global comfort than their female counterparts (32.2 SD 7.7 vs. 28.8 SD 6.0). Similarly, those who knew a family member or person with AIDS scored significantly higher $(\mathrm{P}<0.5)$ on global comfort than those who did not. No significant difference in knowledge, attitude and global comfort was found by specialty, religion, marital status, having or not having children, prior instructions on 
AIDS or mode of the instruction. No significant $(\mathrm{P}>0.05)$ difference was found between the two groups of physicians.

Data was also analyzed to ascertain any link between the sociodemographic variables and scores on knowledge, attitude and global comfort and between knowledge, attitude and global comfort.
Significant but tenuous correlation was found between attitude and age $(\mathrm{r}=.23, \mathrm{P}<.01)$, and between knowledge and attitude $(\mathrm{r}=.21, \mathrm{P}<.05)$. Modest correlation was found between attitude and global comfort $\quad(\mathrm{r}=0.37, \quad \mathrm{P}<0.002)$. Previous AIDS instruction did not correlate with knowledge, attitude or global comfort.

Table 3: Physicians' knowledge, attitude and level of comfort based on their demographic and previous encounter with patients living with AIDS

\begin{tabular}{llll}
\hline Variable & Yes & No & P value \\
& Mean (SD) & Mean (SD) & \\
\hline
\end{tabular}

$\begin{array}{llll}\text { Knowledge about AIDS } & 26.8(2.3) & 27.1(2.9) \\ \text { Have children } & 27.1(2.6) & 26.2(2.9) & \\ \text { Ever been asked to provide services } & & & \\ \text { to PWA } & 26.9(2.7) & 27.9(2.5) \\ \text { Ever cared for PWA } & 26.2(1.8) & 27.0(2.7) \\ \text { Ever refused to provide services to PWA } & 27.0(2.7) & 26.9(2.6) \\ \text { Know of family member or PWA } & 27.2(2.7) & 25.9(2.3)^{\wedge} & \\ \text { Wiling to care for PWA } & 27.0(2.6) & 26.4(1.8) \\ \text { Received instructions on AIDS } & & & \\ & & & \\ \text { Attitude towards AIDS } & 111.6(23.4) & 110.2(21.6) & 0.0034 \\ \text { Have children } & 112.4(21.6) & 93.0(21.1) & \\ \text { Ever been asked to provide services } & 110.8(22.5) & 108.8(22.5) & \\ \text { to PWA } & 96.0(27.9) & 111.2(22.0) & \\ \text { Ever cared for PWA } & 110.5(22.7) & 110.5(21.4) & 0.0060 \\ \text { Ever refused to provide services to PWA } & 113.5(22.0) & 98.4(18.8)^{\wedge} \\ \text { Know of family member or PWA } & 110.8(22.9) & 107.2(14.7) & \\ \text { Willing to care for PWA } & & & \\ \text { Received instructions on AIDS } & 30.9(6.4) & 31.9(8.1) & \\ & 31.9(7.6) & 27.8(4.6) & \\ \text { Global Comfort in Providing Care to PWA } & & & \\ \text { Have children } & 31.7(7.6) & 29.6(5.5) & \\ \text { Ever been asked to provide services } & 29.5(5.2) & 31.6(7.5) & 0.0452 \\ \text { to PWA } & 32.5(8.2) & 29.9(5.9) \\ \text { Ever cared for PWA } & 32.5(7.4) & 26.7(6.1)^{\wedge} & 0.0019 \\ \text { Ever refused to provide services to PWA } & 31.3(7.8) & 32.6(4.3) & \\ \text { Know of family member or PWA } & & \end{array}$

PWA denotes patients or persons (living) with AIDS; ^ Values for "No" responses are for those who answered "No" or "Undecided" ; Means with indicated p-values are significantly different from each other.

\section{Discussion}

\section{Knowledge Attitude and Global Comfort}

Individuals with HIV infection and AIDS have continued to constitute a rapidly growing segment of the society. Of the estimated 38 million people infected with HIV virus worldwide in 2004, more than three-fifth resides in sub-Sahara Africa, and about four million are Nigerians [14]. On the basis of these dismal data and the lack of a cure for the disease, it is critical for medical educators to be familiar with the biases and stereotypes that physicians may have about persons with AIDS. 
Physicians in this study showed satisfactory knowledge on HIV/AIDS. However, they harbored negative attitude and showed low degree of comfort in caring for PWA. The satisfactory knowledge on AIDS corroborates previous studies that reported good or high knowledge level among US, Canadian, Thai, Indian and Chinese physicians [5-8]. A satisfactory knowledge among physicians in the teaching hospitals is positive. However, with $45.5 \%$ of the physicians in the teaching hospitals scoring less than $80 \%$ in the knowledge questions, knowledge deficits among physicians may be more widespread in Nigeria than depicted in the present study. This is because teaching hospitals serve as referral centers to general hospitals and community health centers, which are less likely to be staffed by specialists and physicians with experience in managing conditions with presumed multi-organ systems risk such as AIDS.

Negative attitude shown by the Nigerian physicians affirms those of previous studies of physicians in several countries in North America, Asia and Africa [2,4,5,9]. Poor attitude among physicians more than two decades after AIDS was first discovered, showed that a lot need to be done to change health professionals' attitude. The neutral level of comfort shown by physicians in this study indicates a better degree of comfort than those of Indian and Thai physicians who were uncomfortable sitting at the same table with AIDS patients [5]. On a positive note, many physicians in this study were willing and have never refused care to PWA. This indicates their adherence to the Hippocratic Oaths, which enjoined them to render care to patients regardless of self-interest, even in the face of fear and uncertainty. On a discordant note however, their neutral level of comfort shows that they may hesitate to provide care, or even deny care to PWA.

We found no correlation between knowledge and comfort. This finding may be attributed to a pervasive fear of AIDS in the society and among health professionals [6]. Positive relationship between knowledge and attitude, and between attitude and global comfort indicates that physicians with more knowledge showed more positive attitude. It also indicates that attitude is a crucial link between knowledge and feeling of comfort, and that individuals with positive attitude towards PWA could be more willing to provide care for this population than those with less positive attitude.

\section{Influence of Sociodemographics and Encounter Experience}

Our study showed that male physicians feel more comfortable in providing care to PWA than their female counterparts. This finding does not necessarily mean the female physicians are less understanding or are judgmental about AIDS. Rather, it may be attributed to the societal norms, which expects the male to show less fear and discomfort when faced with uncertainties and unpleasant situation, compared to their female counterparts. Better attitude shown by the consultants and registrars as a group, compared to the group of senior house officers and house officers indicates that negative attitude could be mediated through the course of medical education.

Physicians willing to care for AIDS patients were more knowledgeable, showed better attitude and were more comfortable in caring for PWA than those who were unwilling or undecided. Those who knew a family member or someone with HIV/AIDS were more comfortable than their counterparts who did not know anyone with AIDS. Knowing someone with AIDS could promote increased empathy for PWA and could have mediating effect on physicians' fears of contagion. Those who had previously been asked to provide services to PWA showed better attitude than those who had not been asked. This finding corroborates previous studies that reported better attitude among those with previous AIDS patient care experience $[5,7,15]$, and affirms the importance of practice exposure in improving attitude.

\section{Conclusion}

Nigerian physicians showed satisfactory knowledge on AIDS, but they harbored negative attitude and were neither comfortable nor uncomfortable in providing care to PWA. Previous AIDS care experiences, age and rank influenced physicians' attitude, while male gender and knowing a family member or someone with AIDS influenced their comfort with AIDS patients care. Physicians' knowledge is weakly but positively associated with attitude, while attitude is modestly associated with global comfort in caring for PWA.

This study reinforced the need for ongoing education on AIDS for all physicians, regardless of their practice setting or specialty. Continuing education and medical education curriculum focused on experiential learning, and socialization process honing on skills in generic abilities including empathy, are important in order to improve physicians' attitude and consequently enhance their feeling of comfort in caring for PWA or any contagious disease. Role-playing scenarios on AIDS and guided discussion on psychosocial issues and ethics that could help physicians and trainees in exploring their attitudes and values are suggested as interventions in mitigating prejudices and fear, and in 
preventing HIV related stigma and discriminatory care for PWA.

\section{References}

1. Radecki S, Shapiro J, Thrupp LD, Gandhi SM, Sangha SS, Miller RB. Willingness to treat HIV-positive patients at different stages of medical education and experience. AIDS Patients Care and STDS. 1999;13 :403-14.

2. Feldmann TB, Bell RA, Stephenson JJ, Purifoy FE. Attitudes of medical school faculty and students towards acquired immunodeficiency syndrome. Academic Medicine. 1990; 65: 464-466.

3. Gemson DH, Colombotos J, Elinson J. Fordyce EJ, Hynes M, Stoneburner R. Acquired immunodeficiency syndrome Prevention: Knowledge, Attitudes, and practices of primary care Physicians. Archives of Internal Medicine. 1991; 151:1102-1108.

4. Adelekan ML, Jolayemi SO, Ndom RJ, Adegboye J, Babatunde S, Tunde-Ayimode M, Yusuff O, Makanjuola AB. Caring for people with AIDS in a Nigerian Teaching Hospital: staff attitudes and knowledge. AIDS Care. 1995; 7:S63-73.

5. Brachman $\mathrm{P}$ Jr, Korzarsky $\mathrm{P}$, Cetron M, Jacob MS, Boonit B, Wongsrichanalai J, Keystone JS. Knowledge and Attitude of Hospital -Based Physicians and trainees About HIV Infection in the United States, Canada, India, and Thailand. Archives of Internal Medicine. 1996; 156: 761-766.

6. Yedidia MJ, Berry CA, Barr JK. Changes in physician's attitudes towards AIDS during residency training: a longitudinal study of medical school graduates. Journal of Health and Social Behavior. 1996; 37: 179-191.

7. Fournier PO, Baldor RA, Warfield ME, Frazier B. Patients with HIV/AIDS: physicians' knowledge, attitude, and referral practices. Journal of Family Practice. 1997; 44: 85-9.

8. Buskin SE, Li L, Yin H, Yu T, McGough JP. HIV/AIDS knowledge and attitudes in Chinese medical professionals and students before and after an informational lecture on HIV/AIDS. Journal of Public Health Management and Practice. 2002; 8: 38-43.

9. Fido A, Al Kazemi R. Survey of HIV/AIDS knowledge and attitudes of Kuwaiti family physicians. Family Practice. 2002; 19: 6824.
10. Skiest DJ, Keiser P. Human immunodeficiency virus infection in patients older than 50 years. A survey of primary care physicians' beliefs, practices, and knowledge. Archives of Family Medicine. 1997; 6: 289-94.

11. Held SL. The effects of an AIDS education program on the knowledge and attitudes of a physical therapy class. Physical Therapy. 1993; 73: 156-164.

12. Balogun JA, Kaplan MT, Hoeberlein-Miller T, Anthony A, Lefkowitz R, Hsia L. Knowledge, Attitudes, and willingness of Junior Health Care professional Students to Provide Services for Patients With Acquired Immunodeficiency Syndrome. Journal of Physical Therapy Education. 1998; 12: 5763.

13. Tuckman BW. Conducting Educational $\underline{\text { Research }}\left(2^{\text {nd }}\right.$ ed.). New York: Harcourt Brace Jovanovich Inc., 1978 pp 231-232.

14. WHO/UNAIDS epidemiological facts sheets on HIV/AIDS and sexually transmitted infections 2004 updates. Available from URL:http://www.who.int/hiv Assessed August 30, 2004.

15. Londan BE, Wilson IB, Wenger NS, Cohn SE, Fitchenbaum CJ, Bozzette SA, Shapiro MF, Cleary PD. Specialty training and specialization among physicians who treats HIV/AIDS in the United States. Journal of General Internal Medicine, 2002; 17: 12-22. 